\title{
The design of virtual lower limb rehabilitation for post-stroke patients
}

\author{
Lee Wei Jian, Syadiah Nor Wan Shamsuddin \\ Faculty of Informatics and Computing, Universiti Sultan Zainal Abidin, Malaysia
}

\begin{tabular}{|c|c|}
\hline Article Info & ABSTRACT \\
\hline Article history: & Stroke is one of the leading causes that elicits to disability for adults over a \\
\hline Received Nov 1, 2018 & $\begin{array}{l}\text { long period. Post-stroke patients often have difficulties with joints and muscles } \\
\text { in their legs, which prevents them from moving around. Lower limb }\end{array}$ \\
\hline Revised Feb 6, 2019 & rehabilitation helps to regain normal mobility and functionality of patients \\
\hline Accepted Mar 15, 2019 & $\begin{array}{l}\text { such as standing, walking and climbing stairs. The implementation of virtual } \\
\text { reality in stroke rehabilitation helps to encourage patients on frequent }\end{array}$ \\
\hline Keywords: & $\begin{array}{l}\text { engagement with exercise. This paper briefly presents the ongoing research } \\
\text { regarding lower limb rehabilitation systems for post-stroke patient in virtual }\end{array}$ \\
\hline $\begin{array}{l}\text { Conceptual framework } \\
\text { Lower limb }\end{array}$ & $\begin{array}{l}\text { reality environment to provide an overview of the conceptual design, } \\
\text { limitations, and suggestions for future work in this direction }\end{array}$ \\
\hline
\end{tabular}

Copyright $(2019$ Institute of Advanced Engineering and Science. All rights reserved.

\section{Corresponding Author:}

Lee Wei Jian,

Faculty of Informatics and Computing,

Universiti Sultan Zainal Abidin, Malaysia.

Email: sdusk@live.com

\section{INTRODUCTION}

Stroke is a common disabling condition, which is the second leading cause of death and one of the main causes of acquired adult disability in many countries [1]. Stroke can lead to low levels of physical fitness such as severe disability, partial paralysis, and even death. Stroke survivors often experience limitations in range of motions, fine motor skills, gross motor skills, reaching, and manipulation. Furthermore, common impairments after stroke such as post-stroke pain may compromise one's capacity to carry out activities of daily living (ADL) tasks [2]. This causes the patient to be more dependent on others in doing personal management such as feeding, driving and ADL tasks that may lead to depression and social isolation in life. Problems with joints and muscles in lower limb reduce the efficiency during a walk. Post-stroke patient usually suffers from gait dysfunction, which involves the hyperextension of the knee during the support phase and the reduction of ankle dorsiflexion during hill contact [3]. This causes excessive energy cost during walking, decrease gait efficacy and may increase the risk of falls from the patients.

Virtual reality (VR) describes the stimulation of multiple sensory modalities such as visual, auditory or haptic experiences through the interactions between an individual and a computer-generated environment [4]. VR technology provides an immersive, multisensory, and three-dimensional (3D) environment that is similar to the real world for the users, enabling them to have modified experiences of reality.

In the medical sector, virtual rehabilitation utilizes the exciting features of VR to increase patient's motivation and engage patient in actively taking part in their therapy. This advanced technology also scientifically validated as a supplemental and stand-alone (e.g., pain distraction) treatment option for a variety of pain and behavioral conditions, and it is emerging as a tool for the detection of neurological disorders [5].

This paper briefly explains about VR therapy for lower extremities by (1) identifying the various game elements; (2) designing a conceptual framework for the virtual lower limb rehabilitation after stroke. This will help guide future research in rehabilitation using VR. 


\section{CONCEPTUAL FRAMEWORK}

The conceptual framework was proposed for designing virtual lower limb rehabilitation in virtual environment. The combination of learning theories and gamification approach is as shown in Figure 1. Three theories of motivation: Self Determination Theory (SDT), operant conditioning, and theory of influence in our virtual rehabilitation to enhance the patient's motivation and encourage their engagement in rehabilitation. The application of Mechanics Dynamics Aesthetics (MDA) framework and GameFlow model are aimed at developing a prototype that ensures more effective rehabilitation process without affecting the patient's enjoyment. In our design, patients will perform leg-strengthening exercises (sitting knee extension and sitting to standing) based on the game level selected. Due to the high accessibility of markerless motion sensor, Microsoft Kinect is chosen as the sensor to capture kinematic data. These data are calculated using vector calculus to obtain the knee angle of the patient. Data are stored in a database for the assessment and evaluation of the patient's performance.

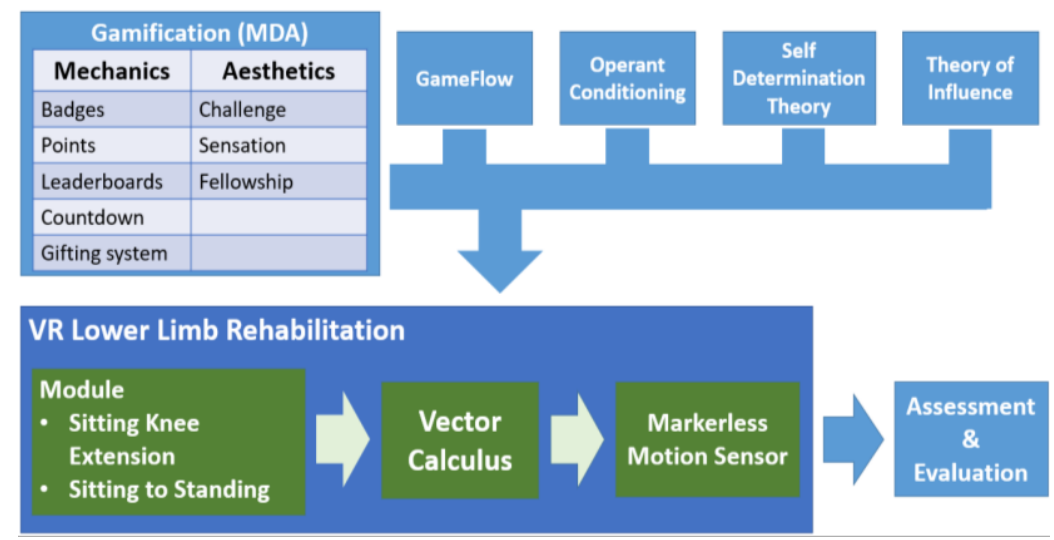

Figure 1. Conceptual framework

\section{THEORIES OF MOTIVATION}

Motivation is an important aspect of rehabilitation, which will affect one's performance and productivity. The incorporation of motivational theories will encourage the engagement of patient and causes the rehabilitation process to be more effective. Three theories of motivation are selected for this purpose: SDT, operant conditioning, and theory of influence.

\subsection{Self Determination Theory (SDT)}

SDT is a theory of human motivation that proposes human are organisms with intrinsic psychological growth and developmental potential. There are two basic motivation categories: intrinsic and extrinsic motivation, based on the different reasons or goals that result in a certain behavior [6]. Extrinsic motivation refers to the obtaining of a certain outcome from the external environment or stimuli such as gaining a high score or avoiding punishment, which can be used to enhance the behavior of an individual. Intrinsic motivation involves the execution of an action with inherent satisfaction, interest, and enjoyment in the task itself, existing within the individual without the desire for any external pressure or reward. Competence, Relatedness, and Autonomy are the three intrinsic psychological needs that allow optimal growth and leads to intrinsic motivation upon satisfaction [7]. Competence states that the motivation of a person increases when he/she feels competent for the action executed. Relatedness explains that the establishment of close relationships with others influences motivation due to the feeling of belongingness and connectedness. Autonomy describes that people are more motivated when they are under one's control and spontaneous behavior. Based on SDT, the existence of social element, achievable goals, and freedom in making decisions in the framework can encourage motivation and engagement during the rehabilitation process.

\subsection{Operant Conditioning}

Operant conditioning is one of the two types of conditioning in behaviorism theory. Skinner coined the concept of operant conditioning through his original experiment, which states that all behaviors are motivated by rewards [8]. Operant conditioning involves the reshaping of an individual by providing reward structure through reinforcement schedules to develop specific controlled behaviors [9]. The essence of operant conditioning is that reinforcement or reward increases the frequency of behavior whereas punishment decreases 
its frequency [8]. Various game mechanisms such as power-ups, boosters, and game leveling are meant to induce and reinforce correct behavior, whereas punishments such as losing game lives or player inventory to obstruct incorrect behavior [9].

In this research, we intend to reward patient with items drop, points, and badges for correct behavior such as achieving higher knee extension angle and frequency. Players tend to play harder if they are sure that something favorable or captivating is going to happen.

\subsection{Theory of Influence}

Theory of Influence [10] states how someone can be influenced by relying on six different psychological principles, namely: reciprocity, scarcity, authority, consistency, liking and social proof.

\subsubsection{Reciprocity, liking, and scarcity}

Reciprocity reflects the fact that people tend to give what they received and return the favor. Liking is about the appreciation of people towards those who like and praise them, which creates an assumption of trustworthiness and kindness in every later encounter. Scarcity relates to the desire for scarce things such as unique and exclusive rewards, which becomes a motivation aspect through the fear of losing the potential interest of the scarce element. According to these elements, we suggest implementing rare item drops for gifting system to promote social interaction between the patients.

\subsubsection{Authority}

Authority states that people usually respect authority and pay attention to those with more experience and having good recommendations such as doctors. The patient should be more confident and willing to participate in rehabilitation in the presence of an expert. Expose the characteristics of an expert such as wearing a lab coat during rehabilitation utilize this principle.

\subsubsection{Consistency}

Consistency explains that people are more willing to be committed when these commitments are public, active, and voluntary. The patient can choose between levels, allowing them to commit voluntarily to the rehabilitation process.

\subsubsection{Social Proof}

Social proof is about how people imitate and follow the lead of similar others. Human beings dependent heavily on the people around them to think, feel, and act [10]. People feel more connected and being part of a group if they are provided with feedback about what others are doing. He/She will be informed about others' progress through the leaderboards.

\section{GAMIFICATION APPROACH}

Gamification is used to increase motivation through the implementation of game design elements in non-game context. GameFlow model and MDA framework are embedded in this conceptual framework.

\subsection{GameFlow}

The GameFlow model is consists of eight elements: concentration, challenge, skills, control, clear goals, feedback, immersion, social interaction, which is used to design, evaluate and understand enjoyment in games [11].

1) Concentration states that games should require concentration and player can maintain focus throughout the game without burdened with unimportant tasks. There should be a high amount of workloads but does not exceed the players' perceptual, cognitive, and memory limits.

2) Challenge element in games should be sufficiently challenging and match the player's skill level at an appropriate pace. Three different level: initial, intermediate, and expert levels support the development of player skills without reading the manual and feel boring.

3) Players should have a sense of control over the game through the game interface and input devices such as start, save and stop. Freedom of making decision applies when patients are free to choose their favorite level.

4) The presentation of goals should be clearly and appropriately to avoid confusion. Visible and distinctive icons are designed to distinguish interactable icons from others.

5) Feedback describes the need for appropriate notification about the player's progress such as score and status to determine the progress towards objectives. Countdown, score, and angle are displayed during the gameplay for patients to adjust their rhythm for achieving the objective. 
6) Immersion states that elements such as audio and narrative can affect players' senses and draw them deeper into the game, where they are emotionally involved in the game and become less aware of their surroundings. Virtual reality is used in this conceptual framework to attract player's attention.

7) Social interaction such as discussion, cooperation, and competition is a strong element of enjoyment in games, as people play games for social interaction; even they dislike game [11]. The patient feels appreciated and belongs to a group when others gift them. Gifting system also enables the patient to return the favor, allowing a continuous social interaction established in the game.

GameFlow model acts as a guideline to develop basic requirements for the prototype.

Table 1. Summarized Features Based on GameFlow model [11]

\begin{tabular}{ll}
\hline \multicolumn{1}{c}{ Elements } & \multicolumn{1}{c}{ GAME FEATURES } \\
\hline Concentration & Focus on one limb per game session \\
Player skills & Multiple levels with different difficulties \\
Control & Start, save and stop game functions \\
Clear goals & Visible and distinctive icons \\
Feedback & Display timer, score, and angle during gameplay \\
Immersion & Virtual reality approach \\
Social interaction & Gifting system \\
\hline
\end{tabular}

\subsection{MDA}

MDA framework is consists of three major concepts: Mechanics, Dynamics, and Aesthetics [12].

\subsubsection{Aesthetics}

Aesthetics describe the desirable emotional responses induced in the player during the interaction with the game system [12]. The interaction of players and the game system enhance their motivation and engagement through the stimulation of emotional responses such as fun and surprise while feeling satisfied upon task completion. However, game elements that are too challenging might depress and decrease the user experience, which will reduce the player's productivity [13]. Game aesthetics is consists of the following without the limitation of taxonomy [12]:

a. Sensation: Game as sense-pleasure

b. Fantasy: Game as make-believe

c. Challenge: Game as obstacle course

d. Fellowship: Game as social framework

e. Expression: Game as self-discovery

f. Submission: Game as pastime

Three aesthetics: challenge, sensation, and fellowship are selected to integrate into the conceptual framework. According to previous study [14], rehabilitation games must be challenging enough along with appropriate console interface to attract the attention of elderly users for active participation. Rehabilitation game with appropriate level of challenge for the patient's current skill level keeps the patient engaged, thus encourage the patient's skill development [9]. The application of sensation element in virtual game design can stimulate the brain of the elders and achieve the purpose of moderate exercise [16].

\subsubsection{Dynamics}

Game dynamics are the run-time behavior of the mechanics based on player inputs and each other's outputs over time [12]. Dynamics form the reason behind the user's motivational behavior towards the game mechanics, which work to create aesthetic experiences. It usually takes a few mechanics to form a game dynamic. For example:

a. Time pressure and opponent play can create challenge.

b. Fellowship is about the team play and social interaction such as sharing information or supplying winning conditions that are more difficult to achieve alone.

Game dynamics that are expected based on the selected aesthetics are as shown below:

a. Competition between players encourages the frequent involvement in the rehabilitation process.

b. Virtual environment can create immersive gameplay to attract the player's attention.

c. Gifting system allows the social interaction between the players without chat by gifting each other. 


\subsubsection{Mechanics}

Mechanics is described as the particular game components at the level of data representation and algorithms, which have a strong impact on the player's motivation and engagement [12]. It is also referred to as the various actions, behaviors, and control mechanisms sustainable by the player within a game context [17]. Points, badges, leaderboards, levels, quests, countdowns, missions, and other particular rules and rewards are the common game mechanics used by game developers.

Based on the discussion earlier, game mechanics are determined and summarized below. Player will feel more challenging with the presence of game mechanics such as achievements, leaderboards, and points. Besides, having the ability to switch between game levels and collect rare item drops creates immersive gameplay, which will stimulate the sensation of the player. Gifting system encourages the social interaction between the players by gifting each other. Table 2 shows the MDA components derived.

Table 2. MDA Components derived [12]

\begin{tabular}{|c|c|c|c|}
\hline Theories of Motivation & MECHANICS & Dynamics & Aesthetics \\
\hline Competence & Achievements & $\begin{array}{l}\text { Earn game items for } \\
\text { character development }\end{array}$ & Challenge \\
\hline Social proof & Leaderboards & $\begin{array}{l}\text { Opponent play and } \\
\text { time pressure }\end{array}$ & Challenge \\
\hline Operant conditioning & $\begin{array}{l}\text { Points } \\
\text { (distance/height) }\end{array}$ & Collect points & Challenge \\
\hline Scarcity & Item drops (gifts) & Collect gifts & Sensation \\
\hline Consistency Autonomy & Different game levels & Change levels/worlds & Sensation \\
\hline $\begin{array}{l}\text { Reciprocity } \\
\text { Liking } \\
\text { Relatedness }\end{array}$ & Gifting system & $\begin{array}{l}\text { Gift or receive gift } \\
\text { from others }\end{array}$ & Fellowship \\
\hline
\end{tabular}

\section{KINEMATIC DATA CAPTURING}

Real-time motion data such as knee angle is captured during the knee extension phase. Data are collected using a motion sensor and processed before further use.

\subsection{Vector Calculus}

Joint angles can be calculated by using the dot product of vectors [18]. $\theta_{\text {Hip }}$ is the angle between vector $\mathrm{EF}$ and vector of hip center point, $\theta_{\text {Knee }}$ is the angle between vector $\overrightarrow{E F}$ and vector $\overrightarrow{F G}, \theta_{\text {Ankle }}$ is the angle between vector $\overrightarrow{F G}$ and vector $\overrightarrow{G H}$. The formula for dot product of vectors is used by assuming that the angle between vector $\vec{a}$ and vector $\vec{b}$ is $\theta$.

$$
\theta=\arccos \left(\frac{\vec{a} \cdot \vec{b}}{|\vec{a}||\vec{b}|}\right)
$$

The coordinates of the hip, knee, and ankle joints are needed to calculate the angle at the knee joint using the dot product of the vector [19]. Figure 2 shows the calculation of joint angle or joint between two connected vectors to evaluate the actual movement of the patients [20].

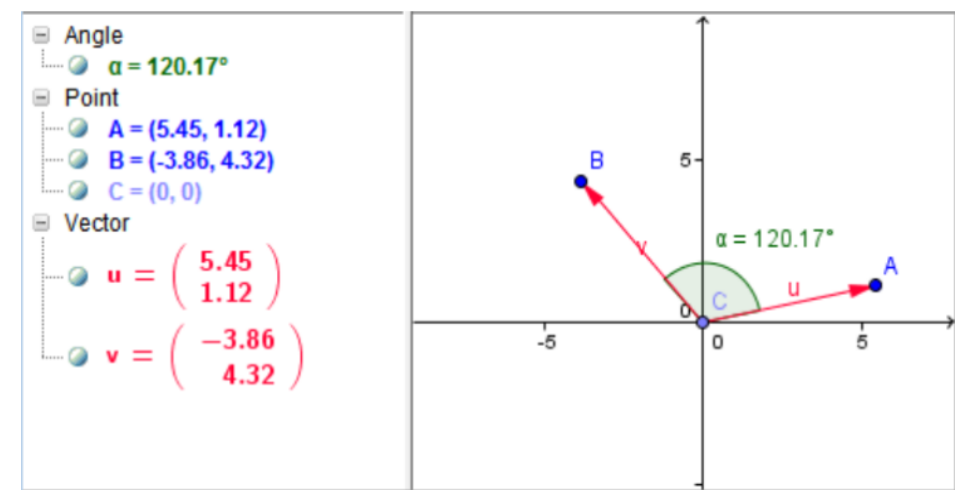

Figure 2. Joint angle calculation [20] 


\subsection{Motion Sensor}

Microsoft Kinect was chosen as the markerless motion sensor due to its capability of leading user into an immersive virtual reality rehabilitation without having to wear any gears. It is important to obtain accurate joint angle to determine the patient's progress. Hence, we have selected the vector calculus as the joint calculation technique since it only involves simple mathematical calculation.

\section{DISCUSSION}

The purpose of this research is to adapt the theories and design a conceptual framework, which will be used in the development of prototype for virtual lower limb stroke rehabilitation. The patients use the application where they can perform their rehabilitation in a virtual environment and therapist can assess and evaluate their progress.

Figure 3 shows the login interface. Patient and therapist are required to login before entering into the main menu as shown in Figure 4 and 5. Patients can choose to play 3 of the game available or enter the achievements interface as shown in Figure 4. The concept of autonomy applies here where patients are free to make decisions and have control over the game.

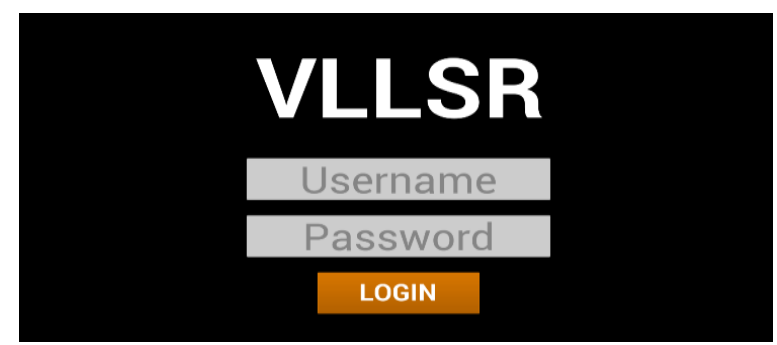

Figure 3. Login Interface

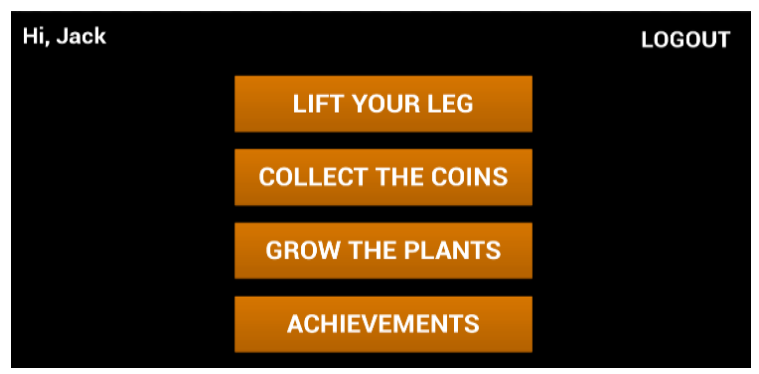

Figure 4. Main menu for patient

Figure 5 is the main menu for therapists where they can choose to register new patient into the virtual rehabilitation or view progress report for patients. The motion data collected are stored in database and therapists can assess and evaluate the patient's progress. This reduces the workload and human error as the data are captured in real time.

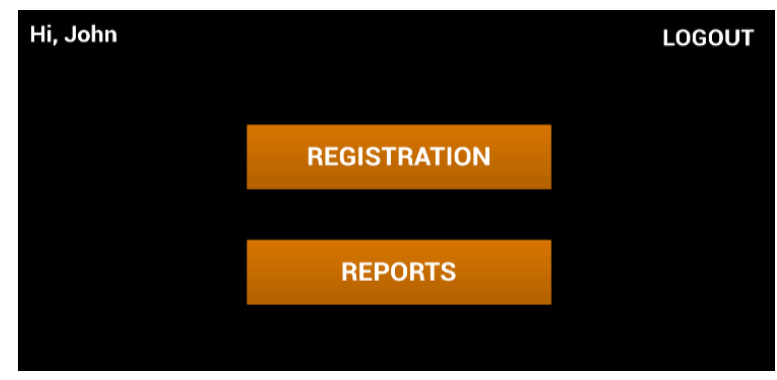

Figure 5. Main menu for therapist 
Figure 6 shows the achievements interface where patients can check for the badges they have earned. Badges are rewarded for each achievement achieved to fulfill the concept of operant conditioning. Achieving a particular achievement will earn one star; each achievement can get a maximum number of three stars. As patients are collecting badges by completing certain challenging requirements, the element of scarcity is fulfilled.

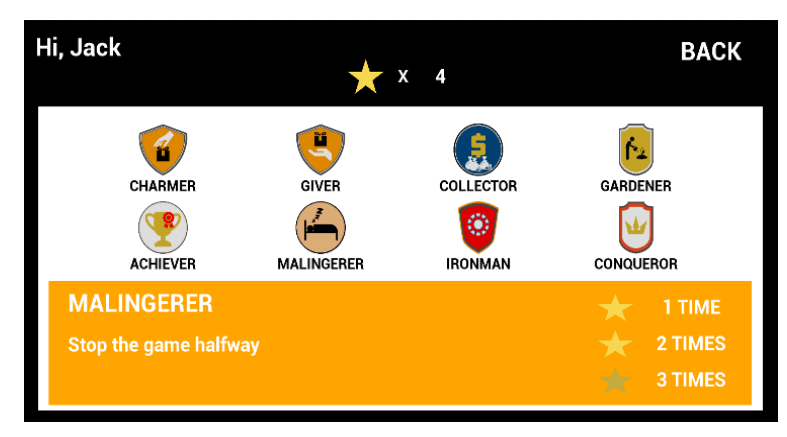

Figure 6. Achievements interface

After choosing a game, patients will enter the menu as shown in Figure 7. The items dropped that can be gifted to others are displayed. Patients can choose to play the game, read the instructions or enter the leaderboards.

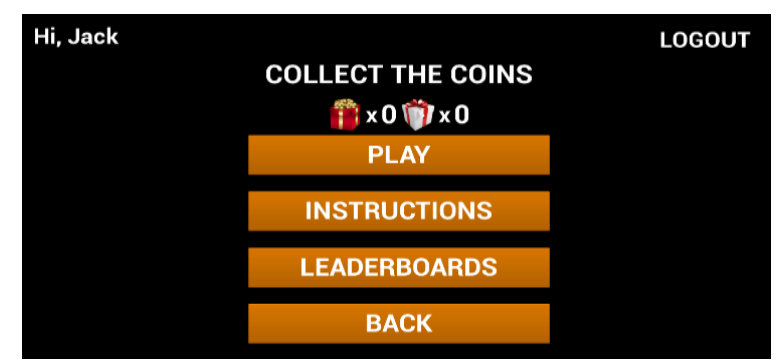

Figure 7. Collect the coins menu

Figure 8 shows the leaderboards. The high scores are displayed for both legs and can be sorted by left or right leg. The gifting system enables others to gift those made it into the leaderboards. The ranking for receiving and gifting are also available in this interface.

Before starting a game, patients are required to choose the left or right leg before proceeding to the gameplay as shown in Figure 9. Figure 10 shows the lift your leg gameplay, where patients can extend both left and right knee simultaneously. The avatar provides real time feedback with the movement performed by the patients.

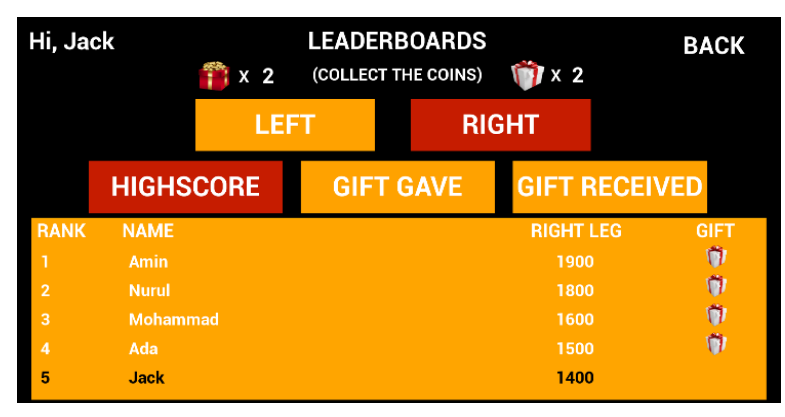

Figure 8. Leaderboards 


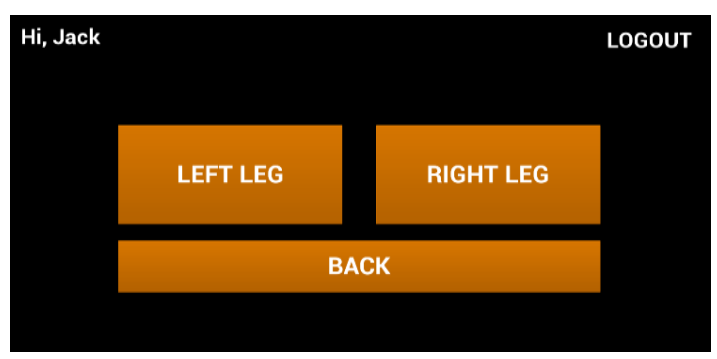

Figure 9. Collect the coins play options

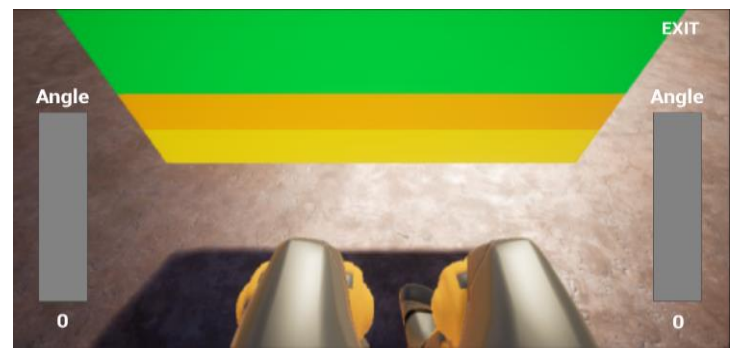

Figure 10. Lift your leg

Figure 11 shows the gameplay for collect the coins. In this game, patients can control the flight altitude by extending their knee angle. They are required to collect as many coins as possible in a limited time.

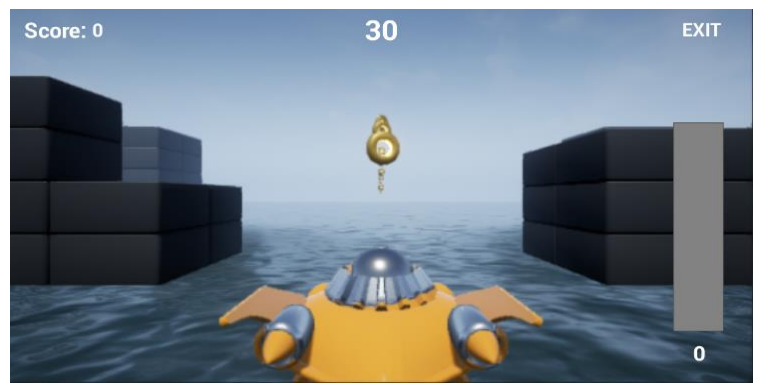

Figure 11. Collect the coins

Grow the plants is the gameplay shown in Figure 12, where patients are required to grow the plants in a limited time. The more plants they grow, the higher the score they can get.

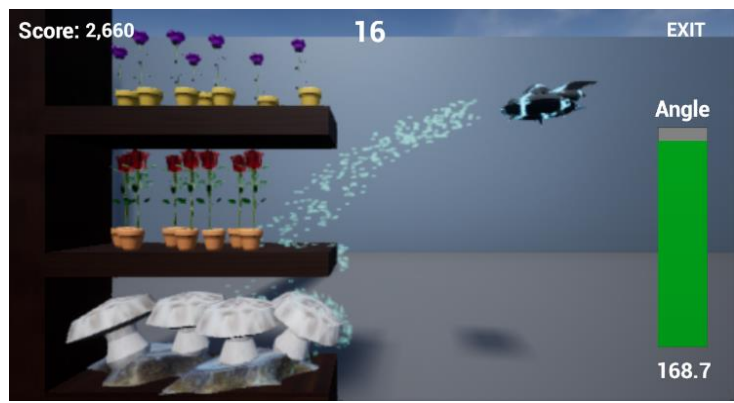

Figure 12. Grow the plants 


\section{CONCLUSION}

This paper presents our ongoing research regarding virtual lower limb rehabilitation for post-stroke patients and the design that our research has rendered. We discuss the importance of motivation, gamification and the need for the development of virtual rehabilitation.

All the game elements discussed are based on previous research and various theories. Future work should be done to determine the suitability of game elements discussed that are included in the rehabilitation game for post-stroke patient. This is to motivate the user accurately and hence, maximize the effect of the therapy.

\section{ACKNOWLEDGMENT}

The authors would like to thank the Government of Malaysia for funding this research under the MOSTI ScienceFund Project (06-01-18-SF0010/RR220) and also the Research Management, Innovation and Commercialization of Universiti Sultan Zainal Abidin.

\section{REFERENCES}

[1] Katan M, Luft A. Global Burden of Stroke. "Seminars in Neurology”, 38(2): 208-211. 2018

[2] Lo Buono V, Corallo F, Bramanti P, Marino S. Coping strategies and health-related quality of life after stroke. "Journal of Health Psychology", 22(1): 16-28. 2016

[3] Aqueveque P, Ortega P, Pino E, Saavedra F, Germany E, Gómez B. “After Stroke Movement Impairments: A Review of Current Technologies for Rehabilitation”, In: Tan U. Editor. Physical Disabilities - Therapeutic Implications. InTech, 96-116. 2017

[4] Cornick JE, Blascovich J. Are Virtual Environments the New Frontier in Obesity Management? "Social \& Personality Psychology", Compass.; 8(11): 650-658. 2014

[5] Wiederhold B, Miller I, Wiederhold M. "Using Virtual Reality to Mobilize Health Care: Mobile Virtual Reality Technology for Attenuation of Anxiety and Pain", IEEE Consumer Electronics Magazine. 7(1): 106-109. 2018

[6] White R. "Motivation reconsidered: The concept of competence", Psychological Review, 66(5): 297-333. 1959

[7] Ryan R, Deci E. Intrinsic and Extrinsic Motivations: Classic Definitions and New Directions. "Contemporary Educational Psychology”, 25(1): 54-67. 2000

[8] Nevid JS. Essentials of Psychology: "Concepts and Applications. Boston: Cengage Learning”, 175-200. 2016

[9] Shah N. "Designing motivational games for robot-mediated stroke rehabilitation. PhD Thesis. Hertfordshire: Postgraduate University of Hertfordshire"; 2016.

[10] Cialdini RB. Influence: "The Psychology of Persuasion”, London: Harper Collins. 1-205. 2009

[11] Sweetser P, Wyeth P. GameFlow: a model for evaluating player enjoyment in games. "Computers in Entertainment", 3(3): 1-24. 2005

[12] Hunicke R, LeBlanc M, Zubek R. MDA: “A Formal Approach to Game Design and Game Research”. Proceedings of the Challenges in Games AI Workshop, Nineteenth National Conference of Artificial Intelligence, 1-4. 2004

[13] Matallaoui A, Hanner N, Zarnekow R. Introduction to Gamification: "Foundation and Underlying Theories". In: Stieglitz S, Lattemann C, Robra-Bissantz S, Zarnekow R, Brockmann T. Editors. Gamification Using Game Elements in Serious Contexts. Cham: Springer, 3-18. 2016

[14] Jie S, Haoyong Y, Chaw TL, Chiang CC, Vijayavenkataraman S. "An interactive upper limb rehab device for elderly stroke patients", Procedia CIRP. Bedford, 60: 488-493. 2017

[15] Shin J, Ryu H, Jang S. "A task-specific interactive game-based virtual reality rehabilitation system for patients with stroke: a usability test and two clinical experiments", Journal of NeuroEngineering and Rehabilitation, 11(32): 1-10. 2014

[16] Liu KY, Huang HL, Chen SM. "Combining narrative and dynamic assessment theory to design a game for the elderly on cognitive function training", Proceedings of the 2017 IEEE International Conference on Applied System Innovation: Applied System Innovation for Modern Technology, ICASI 2017. Sapporo, 1849-1852. 2017

[17] Kim B. Game Mechanics, Dynamics, and Aesthetics. "Library Technology Reports", 51(2): 17-19. 2015

[18] Pei W, Xu G, Li M, Ding H, Zhang S, Luo A. "A motion rehabilitation self-training and evaluation system using Kinect", 13th International Conference on Ubiquitous Robots and Ambient Intelligence. Xian, 353-357. 2016

[19] Choi J, Kang D, Seo J, Kim D, Yang S, Tack G. "The development and evaluation of a program for leg-strengthening exercises and balance assessment using Kinect", Journal of Physical Therapy Science, 28(1): 33-37. 2016

[20] The Chien H, Ha Trang D, Viet Dung N. "Kinect-based virtual training system for rehabilitation", International Conference on System Science and Engineering. Ho Chi Minh, 53-56. 2017. 